\title{
Association between PD-L1 and HPV Status and the Prognostic Value of PD-L1 in Oropharyngeal Squamous Cell Carcinoma
}

\author{
Hae Su Kim, MD ${ }^{1}$ \\ Ji Yun Lee, MD \\ Sung Hee Lim, MD \\ Keunchil Park, MD \\ Se-Hoon Lee, MD ${ }^{1}$ \\ Jong-Mu Sun, MD' \\ Young Hyeh Ko, MD2 \\ Chung-Hwan Baek, MD \\ Young-ik Son, $\mathrm{MD}^{3}$ \\ Han Sin Jeong, $\mathrm{MD}^{3}$ \\ Yong Chan Ahn, MD \\ Min-Young Lee, MD \\ Mineui Hong, MD ${ }^{6}$ \\ Myung-Ju Ahn, MD, PhD'
}

\author{
${ }^{1}$ Division of Hematology-Oncology, \\ Department of Medicine, \\ Departments of ${ }^{2}$ Pathology, \\ ${ }^{3}$ Otorhinolaryngology-Head and \\ Neck Surgery, and ${ }^{4}$ Radiation Oncology, \\ Samsung Medical Center, \\ Sungkyunkwan University \\ School of Medicine, Seoul, \\ ${ }^{5}$ Division of Hematology-Oncology, \\ Department of Internal Medicine, \\ Soonchunhyang University Hospital, \\ Soonchunhyang University \\ College of Medicine, Seoul, \\ ${ }^{6}$ Department of Pathology, \\ Kangnam Sacred Heart Hospital, \\ Hallym University College of Medicine, Seoul, \\ Korea
}

Correspondence: Myung-Ju Ahn, MD, PhD

Division of Hematology-Oncology,

Department of Medicine,

Samsung Medical Center,

Sungkyunkwan University School of Medicine,

81 Irwon-ro, Gangnam-gu, Seoul 06351, Korea

Tel: 82-2-3410-3438

Fax: 82-2-3410-1754

E-mail: silkahn@skku.edu

Received July 13, 2015

Accepted August 13, 2015

Published Online September 15, 2015

\begin{abstract}
Purpose
Oropharyngeal squamous cell carcinoma (OSCC) has been recognized as an immunosuppressive disease. Various mechanisms have been proposed for immune escape, including dysregulation of immune checkpoints such as the programmed cell death 1:programmed cell death-ligand 1 (PD-L1) pathway. We investigated the expression of PD-L1 in human papillomavirus (HPV)-negative and HPV-positive OSCC to determine its prevalence and prognostic relevance.
\end{abstract}

\section{Materials and Methods}

Using immunohistochemistry, 133 cases of OSCC were evaluated for expression of PD-L1. Formalin-fixed paraffin-embedded tumor samples were stained with monoclonal antibody (clone $5 \mathrm{H} 1$ ) to PD-L1. PD-L1 positivity was defined as membrane staining in $\geq 20 \%$ of tumor cells. Correlations between PD-L1 expression and HPV status and survival parameters were analyzed.

\section{Results}

Of the 133 patients, $68 \%$ showed PD-L1 expression, and $67 \%$ of patients were positive for p16 expression by immunohistochemistry. No significant difference in PD-L1 expression was observed between $\mathrm{HPV}(-)$ and $\mathrm{HPV}(+)$ tumors $(61 \%$ vs. $71 \%, \mathrm{p}=0.274)$. No significant difference in age, gender, smoking history, location of tumor origin, or stage was observed according to PD-L1 status. With a median follow-up period of 44 months, older age ( $\geq 65$ years) $(p=0.017)$ and T3-4 stage $(p<0.001)$ were associated with poor overall survival $(O S)$, whereas PD-L1 expression did not affect OS in univariate and multivariate analysis.

\section{Conclusion}

PD-L1 expression was observed in the majority of OSCC patients regardless of HPV status. Further large prospective studies are required to determine the role of PD-L1 expression as a prognostic or predictive biomarker, and clinical studies of immune checkpoint inhibitors in OSCC are warranted regardless of HPV status. 


\section{Introduction}

Human papillomavirus (HPV) has been recognized as a cause of a subset of head and neck squamous cell carcinomas (HNSCC) [1]. HPV-associated HNSCC (HPV-HNSCC) arises most commonly in the oropharynx and the incidence of HPV-related oropharyngeal squamous cell carcinoma (OSCC) (HPV-OSCC) has been increasing despite a decline in tobacco consumption and contrary to a diminishing incidence of cancers at other head and neck sites. In the United States, approximately $40 \%-80 \%$ of OSCCs are caused by HPV and the underlying mechanism is believed to be chronic persistent infection leading to carcinogenesis [2]. Compared with HNSCC associated with smoking and/or alcohol, patients with HPV-OSCC tend to be younger, of a higher socioeconomic status, have a favorable natural history, and respond better to treatment [3]. Although at least 15 types of HPV are thought to have oncogenic potential, the vast majority of HPV-OSCCs are associated with HPV type 16, the same type that leads to HPV-associated anogenital cancers [4]. HPV preferentially targets the highly specialized reticulated epithelium in the lymphoid tissue of the tonsils and the tongue base. HPV integrates its DNA genome into the host cell nucleus, leading to expression of the oncoproteins E6 and E7. The E6 protein induces substantial loss of p53 activity, whereas E7 binds and inactivates the retinoblastoma proteins, which are highly immunogenic and would be expected to induce an antitumor immune response [5].

In HNSCC, various mechanisms have been proposed for immune escape including down regulation of tumor antigen presentation, aberrant regulation of the signal transducer and activator of transcription family, release of immunosuppressive cytokines, and dysregulation of immune checkpoint receptors [6]. Tumor infiltrating lymphocytes (TILs) show high expression of co-inhibitory receptors such as cytotoxic $\mathrm{T}$ lymphocyte-associated antigen 4 and programmed cell death 1 (PD-1), so-called immune checkpoints. A persistent high level of PD-1 expression on antigen-presented CD8(+) cytotoxic T lymphocytes leads to "T cell exhaustion," characterized by impaired effector function and persistent expression of inhibitory receptors. Programmed cell deathligand 1 (PD-L1), also known as B7-H1, is a surface glycoprotein that induces T-cell anergy or apoptosis by binding to PD-1 on T lymphocytes [7]. Clinical trials have reported that inhibition of the PD-1:PD-L1 interaction with antibodies specific for PD-1 or PD-L1 has promising antitumor efficacy in patients with various malignancies including melanoma, non-small cell lung cancer (NSCLC), and HNSCC [8-10].

Recent studies reported that the PD-1:PD-L1 axis is highly related to HPV-positive rather than HPV-negative HNSCC $[11,12]$. PD-1 is expressed on effector T cells in both HPV- positive and -negative tumors, however the level of expression appears to be increased in HPV-positive HNSCC, suggesting that PD-1 expression on cytotoxic $\mathrm{T}$ cells is relevant and may play an important role, particularly in HPV-OSCC $[11,12]$. However, the clinical relevance of PD-L1 expression in OSCC remains unclear. We therefore examined PD-L1 expression on tumor cells in HPV-positive and -negative oropharyngeal cancer and analyzed its association with clinicopathologic characteristics and its prognostic relevance.

\section{Materials and Methods}

\section{Patients}

Patients who met the following criteria were recruited: oropharyngeal cancer arising from tonsil, soft palate, posterior wall of oropharynx, and base of tongue; squamous cell carcinoma; curative intent radiation therapy, concurrent chemoradiation therapy (CCRT), or surgery; no evidence of distant metastasis; available medical records and sufficient tumor tissue for immunohistochemical (IHC) staining of PD-L1 and p16. Formalin-fixed paraffin-embedded (FFPE) tissues from a diagnostic biopsy or surgical specimen were retrieved from the Department of Pathology. Baseline clinicopathologic characteristics including age, sex, smoking history, TNM stage at diagnosis, curative modality, and clinical follow-up data were collected retrospectively from the database at Samsung Medical Center. The study protocol and all related materials were approved by the Institutional Review Board of the institution.

\section{HPV expression and p16 IHC}

An HPV-positive tumor was defined by specific staining of tumor cells for $\mathrm{p} 16$ expression. Strong correlation between overexpression of p16 and HPV expression has been reported in several studies [13]. Tumor p16 expression was evaluated by IHC analysis using a mouse monoclonal antibody (CINtec) and visualized using an immunostainer (Ventana XT, Ventana, Tucson, AZ) and Ultraview Universal DAB Detection Kit (Ventana) [14]. Positive p16 expression was defined as diffuse nuclear and cytoplasmic staining in $70 \%$ or more of the tumor cells as determined by a pathologist (Y.H.K.) [14].

\section{PD-L1 expression by IHC}

PD-L1 expression was evaluated by IHC using the monoclonal anti-human B7-H1 antibody (clone 5H1, a non-com- 
mercial mAb kindly provided by Dr. Lieping Chen's laboratory) as previously described [15]. Briefly, FFPE tumor sections were cut at $4 \mu \mathrm{m}$ and then routinely deparaffinized and rehydrated in xylene and graded ethanol solutions. Antigen retrieval was performed in Tris-EDTA buffer. Sections were washed and incubated for 5 minutes in wash buffer (Dako \#S3006, Dako, Glostrup, Denmark), and then placed on the Autostainer Plus (Dako) and subjected to the following protocol using a CSA II kit (Dako \#K1497). First, the activity of endogenous peroxidase was blocked with endogenous blocking solution, and then the section was washed with wash buffer and incubated for 60 minutes with monoclonal antihuman B7-H1 (clone 5H1) diluted 1:1,000 with antibody diluent containing background reducing components. After washing, the sections were incubated with biotin-conjugated rat anti-mouse IgG1 secondary antibody at a 1:500 dilution. Reaction products were visualized by incubation in 3, 3'-diaminobenzidine for 2 minutes and the slides were counterstained with hematoxylin.

A pathologist (M.H.) who was blinded to the patients' clinicopathologic information assessed the expression of PD-L1. PD-L1 positivity was defined as membrane staining in $\geq 20 \%$ of tumor cells. PD-L1 expression on tumor cells was evaluated using the intensity score and proportion score. The intensity of staining was evaluated according to the following scale: 0 , no staining; 1 , weak staining; 2 , moderate staining; and 3, strong staining. Because most prognostic factors are considered as dichotomized and discontinuous variables, a cutoff point was selected to give the optimal separation between low risk and high risk for overall survival (OS). We initially set several cutoff points for selection of the best value, and then selected $20 \%$ staining as the appropriate cutoff value regardless of intensity score.

\section{Statistical analysis}

The relationship between PD-L1 expression and other patient characteristics, including p16 expression, was evaluated using analysis of variance tests for continuous variables and Pearson's chi-square tests for discrete variables. Association of PD-L1 expression with survival of patients with OSCC was also evaluated. OS was defined as the time from the date of diagnosis to death as a result of any cause or the last follow-up if the patient was alive. Progression-free survival (PFS) was defined as the time from the initial date of treatment to disease progression or the last follow-up if the patient had not progressed. Survival curves were generated using the Kaplan-Meier method, and the difference between the curves according to PD-L1 status was evaluated using the log-rank test. The Cox proportional hazard model was used to assess hazard ratios (HRs) of prognostic factors for OS. All factors showing statistical significance in univariate analyses or clinical significance were included in the multivariate analysis. All p-values are two sided, and a level of 5\% was considered statistically significant. All statistical analyses were performed using SPSS ver. 21 (IBM Co., Armonk, $N Y$ ).

\section{Results}

\section{Patient characteristics}

From October 2002 to September 2013, of a total of 198 patients with OSCC who underwent screening, 133 patients $(67 \%)$ were eligible for the current study. Patient characteristics are described in Table 1 . The median age of patients was 57.5 years (range, 35 to 80 years), 120 patients (90\%) were male, and $51(38 \%)$ were never smokers. Seventy-nine patients (59\%) had stage IV disease at diagnosis, and the most common site of tumor origin was tonsil ( $78 \%$ ), followed by soft palate $(7 \%)$ and base of tongue (3\%). Ninety-two patients $(69 \%)$ underwent curative intent surgery, and 35 (26\%) underwent CCRT.

\section{Prevalence of PD-L1 expression on tumor cell membrane}

Overall, PD-L1 expression was positive in 90 patients $(68 \%)$ and negative in 43 (32\%). PD-L1 expression according to proportion was scored using various cutoff values as absent (no staining) in 15 patients $(11 \%), \geq 10 \%$ in $116(87 \%)$, $\geq 20 \%$ in $90(68 \%), \geq 50 \%$ in $51(38 \%)$, and $\geq 80 \%$ in $23(17 \%)$. PD-L1 expression according to intensity was scored as absent in 15 patients $(11 \%)$, weak in $55(41 \%)$, moderate in $43(32 \%)$, and strong in $20(15 \%)$. The proportion score of PD-L1 expression showed correlation with the intensity score (Pearson coefficiency $\mathrm{r}=0.451, \mathrm{p}=0.01$ ).

\section{Association of PD-L1 expression and clinical character- istics}

No statistically significant association was observed between PD-L1 expression and age, sex, location of tumor origin, or stage (Table 1). In addition, smoking history was not associated with PD-L1 expression in the tumor cell membrane $(\mathrm{p}=0.343)$.

\section{Association of PD-L1 and p16 expression}

A total of 89 patients (67\%) were positive for p16. Patients with 16 positivity had more advanced nodal (N2-3; $62 \%$ vs. $48 \%, \mathrm{p}=0.002$ ) and tumor stage (III-IV; $94 \%$ vs. $73 \%, \mathrm{p}=0.001$ ), 
Table 1. Clinicopathologic characteristics of patients with oropharyngeal squamous cell carcinoma and their relationship with PD-L1 expression

\begin{tabular}{|c|c|c|c|c|}
\hline Characteristic & No. of patients ( $n=133$ ) & PD-L1 (+) (n=90) & PD-L1 (-) $(n=43)$ & p-value \\
\hline Age (yr) & $57.5(35.4-80.1)$ & $57.3(35.4-72.8)$ & $57.7(42.2-80.1)$ & 0.203 \\
\hline$\geq 65 \mathrm{yr}$ & $30(22.6)$ & $17(18.9)$ & $13(30.2)$ & 0.108 \\
\hline \multicolumn{5}{|l|}{ Sex } \\
\hline Male & $120(90.2)$ & $78(86.7)$ & $42(97.7)$ & 0.060 \\
\hline Female & $13(9.8)$ & $12(13.3)$ & $1(2.3)$ & \\
\hline \multicolumn{5}{|l|}{ Smoking } \\
\hline Never smoker & $51(38.3)$ & $37(41.1)$ & $14(32.6)$ & 0.343 \\
\hline Ever smoker & $82(61.7)$ & $53(58.9)$ & $29(67.4)$ & \\
\hline \multicolumn{5}{|l|}{ Location } \\
\hline Tonsil & $104(78.2)$ & $75(83.3)$ & $29(67.4)$ & 0.197 \\
\hline Base of tongue & $4(3.0)$ & $2(2.2)$ & $2(4.7)$ & \\
\hline Soft palate & $9(6.8)$ & $4(4.4)$ & $5(11.6)$ & \\
\hline Oropharynx, NOS & $16(12.0)$ & $9(10.0)$ & $7(16.3)$ & \\
\hline \multicolumn{5}{|l|}{ T stage } \\
\hline T1 & $33(24.8)$ & $18(20.0)$ & $15(34.9)$ & 0.247 \\
\hline $\mathrm{T} 2$ & $74(55.6)$ & $53(58.9)$ & $21(48.8)$ & \\
\hline T3 & $15(11.3)$ & $10(11.1)$ & $5(11.6)$ & \\
\hline $\mathrm{T} 4$ & $11(8.3)$ & $9(10.0)$ & $2(4.7)$ & \\
\hline \multicolumn{5}{|l|}{ N stage } \\
\hline N0 & $22(16.5)$ & $14(15.6)$ & $8(18.6)$ & 0.137 \\
\hline N1 & $35(26.3)$ & $19(21.1)$ & $16(37.2)$ & \\
\hline $\mathrm{N} 2$ & $68(51.1)$ & $50(55.6)$ & $18(41.9)$ & \\
\hline N3 & $8(6.0)$ & $7(7.8)$ & $1(2.3)$ & \\
\hline \multicolumn{5}{|l|}{ AJCC stage } \\
\hline I & $8(6.0)$ & $3(3.3)$ & $5(11.6)$ & 0.100 \\
\hline II & $9(6.8)$ & $6(6.7)$ & $3(7.0)$ & \\
\hline III & $37(27.8)$ & $22(24.4)$ & $15(34.9)$ & \\
\hline IV & $79(59.4)$ & $59(65.6)$ & $20(46.5)$ & \\
\hline \multicolumn{5}{|l|}{ HPV p16 status } \\
\hline Positive & $89(66.9)$ & $63(70)$ & $26(60.5)$ & 0.274 \\
\hline Negative & $44(33.1)$ & $27(30)$ & $17(39.5)$ & \\
\hline \multicolumn{5}{|c|}{ First-line curative modality } \\
\hline Operation & $92(69.2)$ & $60(66.7)$ & $32(74.4)$ & 0.280 \\
\hline CCRT & $35(26.3)$ & $27(30.0)$ & $8(18.6)$ & \\
\hline RT & $6(4.5)$ & $3(3.3)$ & $3(7.0)$ & \\
\hline
\end{tabular}

Values are presented as median (range) or number (\%). PD-L1, programmed cell death-ligand 1; NOS, not otherwise specified; AJCC, American Joint Committee on Cancer; HPV, human papillomavirus; CCRT, concurrent chemoradiation therapy; $\mathrm{RT}$, radiation therapy.

and were more likely to have tonsillar cancer $(89 \%$ vs. $57 \%$, $\mathrm{p}<0.001$ ) than those negative for p16 (Table 2). p16 expression was more frequently observed in tonsillar cancer compared with other oropharyngeal cancers $(79 / 104$ [76\%] vs. $10 / 29$ [34\%], $\mathrm{p}<0.001$ ).

No significant correlation was observed between PD-L1 and p16 expression (63/89 [71\%] for p16-positive and 27/ 44 [61\%] for p16-negative tumors, respectively, $p=0.274)$. In analysis with several cutoff values of PD-L1 expression including proportion score and intensity score, no correlation was observed between PD-L1 and p16 expression. When the analysis was restricted to patients with tonsillar cancer, no association was found between PD-L1 expression and p16 expression $(57 / 79$ [72\%] for p16 positive vs. $18 / 25$ [72\%] for p16 negative, $\mathrm{p}=0.988)$. 
Table 2. Clinicopathologic characteristics of patients with oropharyngeal squamous cell carcinoma and their relationship with HPV expression

\begin{tabular}{|c|c|c|c|}
\hline Characteristic & p16 (-) (n=44) & p16 (+) (n=89) & p-value \\
\hline Age (yr) & $59.0(35.4-80.1)$ & $54.9(41.4-73.5)$ & 0.100 \\
\hline$\geq 65 \mathrm{yr}$ & $14(31.8)$ & $16(18.0)$ & 0.072 \\
\hline \multicolumn{4}{|l|}{ Sex } \\
\hline Male & $42(95.5)$ & $78(87.6)$ & 0.130 \\
\hline Female & $2(4.5)$ & $11(12.4)$ & \\
\hline \multicolumn{4}{|l|}{ Smoking } \\
\hline Never smoker & $14(31.8)$ & 37 (41.6) & 0.276 \\
\hline Ever smoker & $30(68.2)$ & $52(58.4)$ & \\
\hline \multicolumn{4}{|l|}{ Location } \\
\hline Tonsil & $25(56.8)$ & $79(88.8)$ & $<0.001$ \\
\hline Base of tongue & $3(6.8)$ & $1(1.1)$ & \\
\hline Soft palate & $8(18.2)$ & $1(1.1)$ & \\
\hline Oropharynx, NOS & $8(18.2)$ & $8(9.0)$ & \\
\hline \multicolumn{4}{|l|}{ T stage } \\
\hline $\mathrm{T} 1$ & $9(20.5)$ & $24(27.0)$ & 0.220 \\
\hline $\mathrm{T} 2$ & $22(50.0)$ & $52(58.4)$ & \\
\hline T3 & 8 (18.2) & $7(7.9)$ & \\
\hline $\mathrm{T} 4$ & $5(11.4)$ & $6(6.7)$ & \\
\hline \multicolumn{4}{|l|}{ N stage } \\
\hline N0 & $15(34.1)$ & $7(7.9)$ & 0.002 \\
\hline N1 & 8 (18.2) & $27(30.3)$ & \\
\hline N2 & $19(43.2)$ & $49(55.1)$ & \\
\hline N3 & $2(4.5)$ & $6(6.7)$ & \\
\hline \multicolumn{4}{|l|}{ AJCC stage } \\
\hline I & $4(9.1)$ & $4(4.5)$ & 0.001 \\
\hline II & $8(18.2)$ & $1(1.1)$ & \\
\hline III & $9(20.5)$ & $28(31.5)$ & \\
\hline IV & $23(52.3)$ & $56(62.9)$ & \\
\hline \multicolumn{4}{|l|}{ PD-L1 status } \\
\hline Positive & $27(61.4)$ & $63(70.8)$ & 0.274 \\
\hline Negative & $17(38.6)$ & $26(29.2)$ & \\
\hline \multicolumn{4}{|c|}{ First-line curative modality } \\
\hline CCRT & $7(15.9)$ & $28(31.5)$ & 0.155 \\
\hline Operation & $35(79.5)$ & $57(64.0)$ & \\
\hline RT & $2(4.5)$ & $4(4.5)$ & \\
\hline
\end{tabular}

Values are presented as median (range) or number (\%). HPV, human papillomavirus; NOS, not otherwise specified; AJCC, American Joint Committee on Cancer; PD-L1, programmed cell death-ligand 1; CCRT, concurrent chemoradiation therapy; $\mathrm{RT}$, radiation therapy.

\section{Association of PD-L1 expression and survival}

Over a median follow-up period of 44 months (range, 2.8 to 119.3 months), 15 PD-L1-positive patients (17\%) and nine PD-L1-negative patients (21\%) died. The 3-year OS was 85\% for PD-L1-positive and 87\% for PD-L1-negative disease. Median PFS and OS have not yet been reached in the entire cohort. Results of Kaplan-Meier analysis showed no signifi- cant difference in PFS and OS between PD-L1-positive and PD-L1-negative patients ( $\mathrm{p}=0.519$ and $\mathrm{p}=0.625$, respectively) (Fig. 1A and B). In analysis with several cutoff values of PD-L1 expression including proportion score and intensity score, we found no significant difference in PFS and OS. The patients were further divided into p16-positive and p16-negative groups. Even though p16-positive patients had more advanced stage, they tended to show a more favorable PFS, 
A
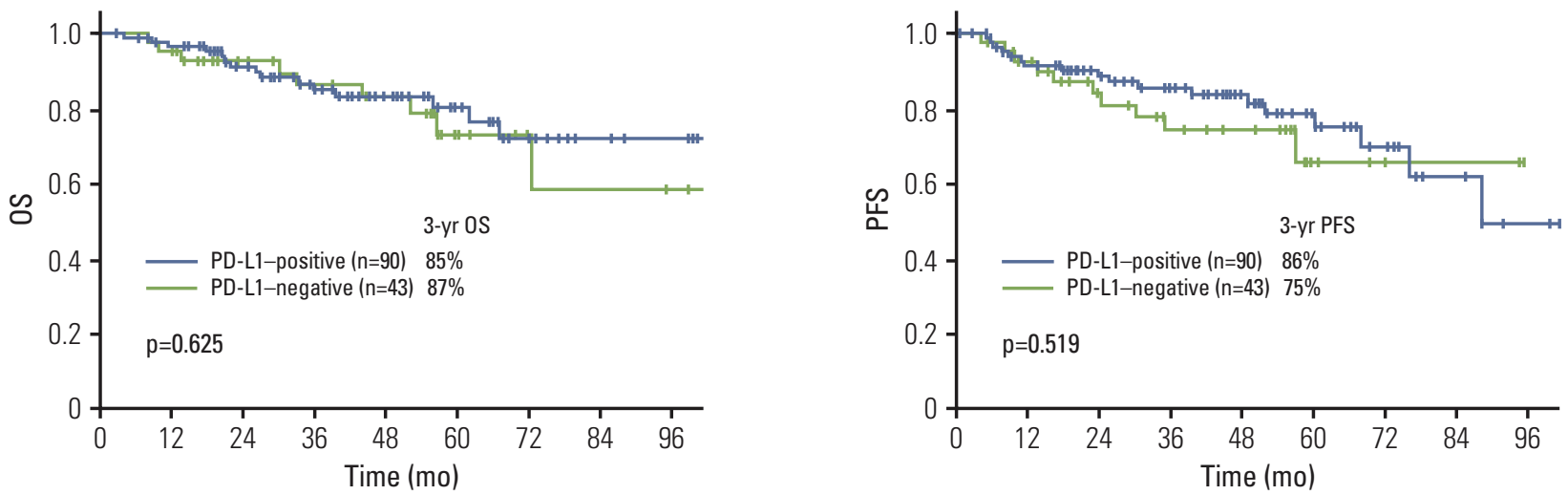

C
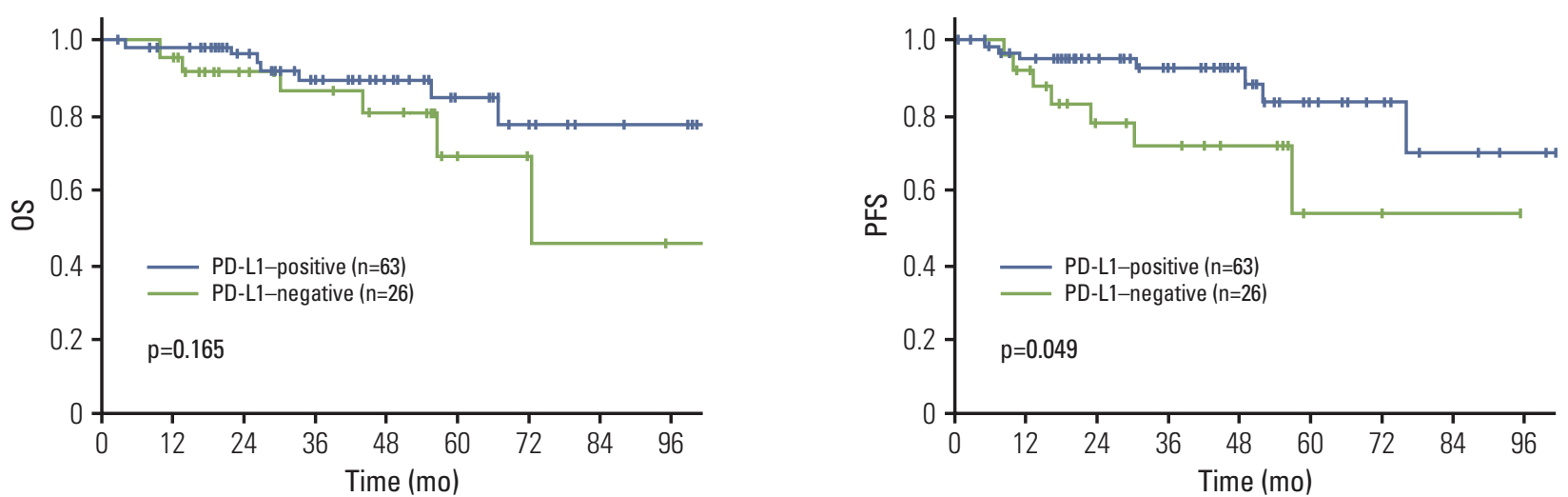

$\mathbf{E}$
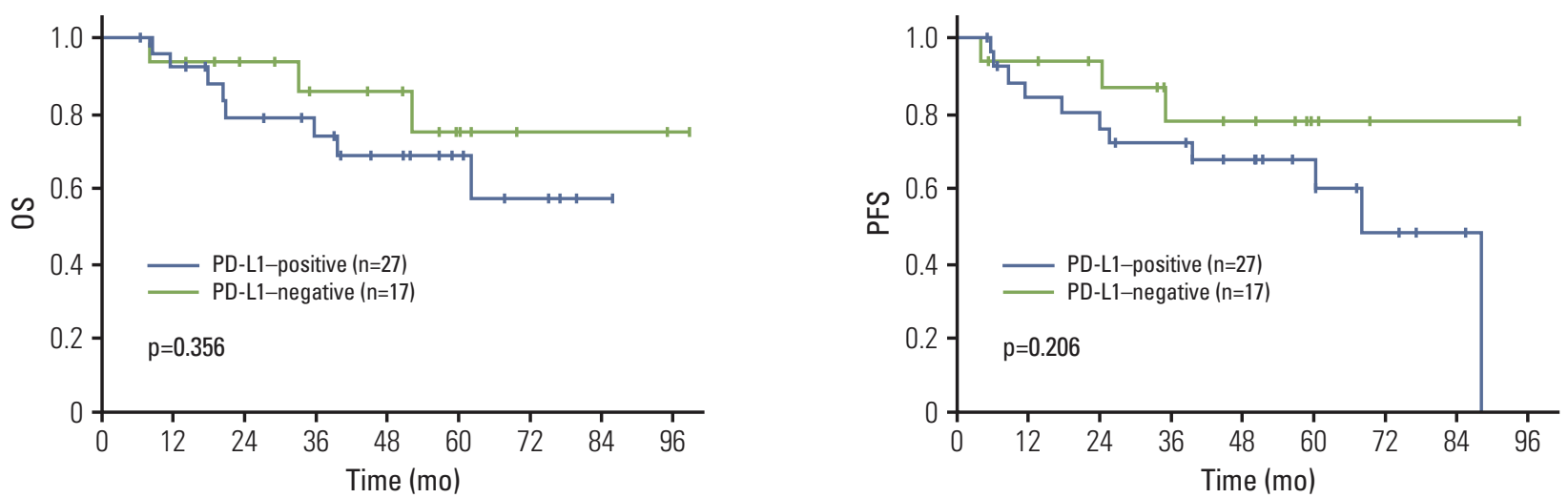

Fig. 1. Kaplan-Meier curves of overall survival (OS) and progression-free survival (PFS) according to programmed cell death-ligand 1 (PD-L1) expression status in patients with oropharyngeal squamous cell carcinoma. The p-value for difference between the two curves was determined using the log-rank test. (A) OS for overall population. (B) PFS for overall population. (C) OS for the p16-positive group. (D) PFS for the p16-positive group. (E) OS for the p16-negative group. (F) PFS for the p16negative group. 

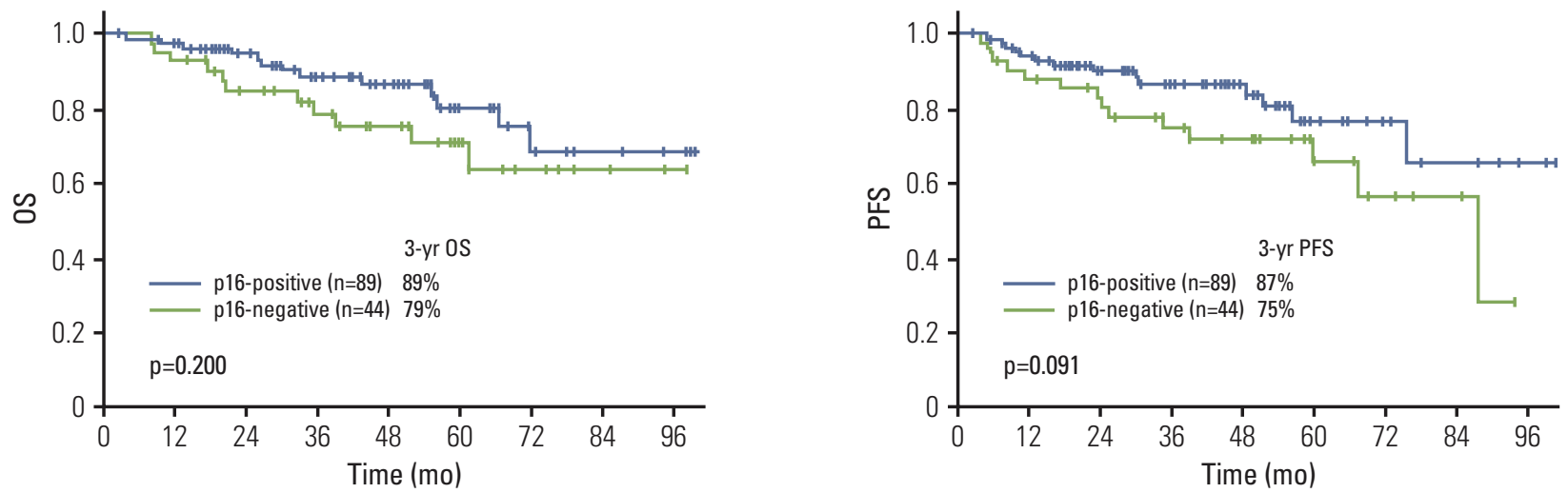

Fig. 2. Kaplan-Meier curves of overall survival (OS) (A) and progression-free survival (PFS) (B) according to p16 expression status in patients with oropharyngeal squamous cell carcinoma.

but without statistical significance ( $\mathrm{p}=0.091)$ (Fig. 2A and B). In the p16-positive subgroup, patients with PD-L1 expression had longer PFS than PD-L1-negative patients $(p=0.049)$, but no significant difference in OS was observed $(p=0.165)$ (Fig. 1C and D). In the p16-negative subgroup, PD-L1 expression was not associated with OS or PFS $(p=0.356$ and $\mathrm{p}=0.206$, respectively) (Fig. $1 \mathrm{E}$ and F).

To determine the prognostic value of PD-L1 expression for OS, univariate and multivariate analyses were performed using the Cox regression model (Table 3). Old age (HR, 2.834; $95 \%$ confidence interval $[\mathrm{CI}], 1.206$ to $6.660 ; \mathrm{p}=0.017)$ and advanced T stage (T1-2 vs. T3-4; HR, 5.806; 95\% CI, 2.430 to 13.868; $\mathrm{p}<0.001)$ remained independent factors for poor prognosis, but PD-L1 expression did not affect OS regardless of HPV status.

\section{Discussion}

HNSCC is recognized as an immunosuppressive disease [16]. In a clinical trial, immunotherapy targeting the PD-1:PD-L1 axis yielded an objective response in a subset of individuals with HNSCC [10] and a recent study suggested correlation of PD-L1 expression in tumors with response to PD-1 targeted therapy [9]. Taking these findings together, it is worth investigating the prognostic role of PD-L1 expression. PD-L1 expression in the tumor has shown correlation with both favorable and unfavorable outcomes in various malignancies. Several studies have examined PD-L1 expression in HNSCC $[11,12,17]$; however, the clinicopathologic characteristics associated with PD-L1 expression remain largely unknown.

In the study by Cho et al. [18], PD-L1 expression was positive in 39 of 43 OSCCs $(91 \%)$ and did not show correlation with clinicopathologic factors or OS. Ukpo et al. [17] reported that PD-L1 was expressed in 84 of 181 patients (46\%) with oropharyngeal cancer and no association was observed between PD-L1 expression and gender, smoking history, stage, or other clinical parameters; however, there was an association between PD-L1 expression and distant metastasis $(\mathrm{p}=0.03)$. In the current study, $68 \%$ of OSCC patients showed PD-L1 expression, consistent with previous results $[17,18]$. However, expression of PD-L1 in tumor cells did not show correlation with any clinicopathologic features, including PFS and OS.

Other studies in nasopharyngeal cancer have reported conflicting results; one study reported an association of PD-L1 expression with TNM stage, while the other showed no correlation with any of the clinicopathologic parameters examined $[19,20]$. These conflicting results in various tumors may be attributed in part to the lack of uniformity in assays, including intra- or inter-observer variability of IHC, lack of standardized antibodies for determining PD-L1 expression, and various cutoff values for defining positive expression.

Another possible explanation is that PD-L1 signaling not only down regulates antitumor effector $\mathrm{T}$ cell immunity, but also interacts with the innate and adaptive immune response [21]. In a recent study, tumors with PD-L1 positivity at baseline showed a significant decrease in PD-L1 expression at disease progression, whereas tumors with PD-L1 negativity at baseline showed a significant increase in PD-L1 expression at disease progression, irrespective of chemotherapeutic reg- 
Table 3. Univariate and multivariate analysis to assess the association of clinical parameters with survival

\begin{tabular}{|c|c|c|c|c|}
\hline \multirow{2}{*}{ Characteristic } & \multicolumn{2}{|c|}{ Univariate analysis } & \multicolumn{2}{|c|}{ Multivariate analysis } \\
\hline & HR $(95 \%$ CI $)$ & p-value & HR $(95 \% \mathrm{CI})$ & p-value \\
\hline Age (< 65 yr vs. $\geq 65$ yr $)$ & $2.531(1.105-5.793)$ & 0.028 & $2.834(1.206-6.660)$ & 0.017 \\
\hline Sex (male vs. female) & $0.042(0.001-10.401)$ & 0.278 & - & - \\
\hline Smoking (never vs. ever) & $2.198(0.904-5.349)$ & 0.083 & $2.270(0.913-5.644)$ & 0.078 \\
\hline Tumor location (tonsil vs. others) & $1.798(0.766-4.220)$ & 0.17 & - & - \\
\hline T stage (T1-2 vs. T3-4) & $6.311(2.656-14.991)$ & $<0.001$ & $5.806(2.430-13.868)$ & $<0.001$ \\
\hline N stage (N0-1 vs. N2-3) & $2.532(1.004-6.384)$ & 0.049 & $2.118(0.836-5.365)$ & 0.114 \\
\hline HPV status (no vs. yes) & $0.595(0.266-1.328)$ & 0.205 & - & - \\
\hline PD-L1: cutoff 5\% (no vs. yes) & $0.698(0.238-2.046)$ & 0.513 & - & - \\
\hline PD-L1: cutoff $20 \%$ (no vs. yes) & $0.814(0.356-1.863)$ & 0.626 & - & - \\
\hline $\begin{array}{l}\text { First-line curative modality } \\
\text { (CCRT / RT vs. operation) }\end{array}$ & $1.365(0.506-3.681)$ & 0.539 & - & - \\
\hline
\end{tabular}

$\mathrm{HR}$, hazard ratio; CI, confidence interval; HPV, human papillomavirus; PD-L1, programmed cell death-ligand 1; CCRT, concurrent chemo-radiation therapy; RT, radiation therapy.

imens [22]. This study suggests the possibility of dynamic changes in PD-L1 expression. Moreover, differences in PD-L1 expression between tumor cells and tumor infiltrating inflammatory cells may be a possible cause of conflicting results. Bhandari and Siva [23] reported that higher PD-L1 expression in tumor cells was associated with advanced disease in patients with urothelial carcinoma and also showed correlation with poor survival. Another study reported that PD-L1 in urothelial carcinoma cells was not predictive for OS, but positive PD-L1 expression in tumor infiltrating mononuclear cells showed significant association with longer survival [24]. Therefore, the prognostic value of PD-L1 expression should be further explored.

HPV-HNSCC arises from deep crypts within the lymphoid tissue of the tonsil and base of the tongue and the majority of cases can be distinguished from HPV-negative HNSCC by the characteristic infiltration of lymphocytes in the stroma and tumor nests [25]. There is substantial evidence that HNSCC is an immunosuppressive disease, particularly HPV-positive HNSCC, and recent preclinical and clinical studies suggest that the PD-1:PD-L1 pathway is highly related to the pathogenesis of HPV-HNSCC $[11,12,16]$. However, it is unclear whether the PD-1:PD-L1 pathway plays a greater role in HPV-positive HNSCC compared to HPV-negative HNSCC.

In the current study, $67 \%$ of patients had p16-positive disease indicating HPV-OSCC, consistent with previous reports [4]. We found that HPV-OSCC was associated with more advanced $\mathrm{N}$ stage, a trend for less advanced T stage, and occurrence at a relatively young age. In addition, there was a trend toward favorable PFS in p16-positive patients compared to p16-negative patients despite the more advanced stage in the p16-positive group. In this study, the small difference in survival benefit in the HPV-positive OSCC group may be attributed to the limited sample size and relatively few events in the entire cohort.

We found no association between PD-L1 expression and HPV status. Similarly, Badoual et al. [11] reported that 33 of 64 HNSCCs $(51.5 \%)$ expressed a significant level of PD-L1 in tumor cells, but no correlation was observed between PD-L1 expression and HPV status (HPV positive vs. negative, $62.5 \%$ vs. $40 \%, \mathrm{p}=0.08$ ). However, HPV-HNSCC was more heavily infiltrated by $\mathrm{CD} 8+\mathrm{T}$ cells $(\mathrm{p}=0.01)$ and $\mathrm{PD}-1+\mathrm{CD} 4+$ $T$ cells $(p=0.045)$, and the total number of PD- $1+C D 4+$ and PD- $1+C D 8+T$ cells $(p=0.045)$ was higher than that in HPVnegative HNSCC. Ukpo et al. [17] reported the presence of PD-L1 expression in 68 of $138 \mathrm{HPV}$ mRNA-positive patients (49\%) compared with 14 of 41 HPV mRNA-negative patients (34\%) (odds ratio, 3.4; $\mathrm{p}=0.08$ ). In contrast, Lyford-Pike et al. [12] reported that PD-L1 was expressed at a much higher level in HPV-positive tumors compared with HPV-negative tumors (14/20 [70\%] vs. 2/7 [29\%]).

A recent study reported that PD-L1 expression in tumors showed correlation with improved efficacy of the immune checkpoint inhibitor anti-PD-1 antibody in NSCLC [9]. The predictive role of PD-L1 expression in HNSCC patients treated with immune checkpoint inhibitors should be further explored. In addition, given the high expression of PD-L1 regardless of HPV status, clinical studies with anti-PD-1 or anti-PD-L1 antibody are warranted in both HPV-positive and HPV-negative patients. Identification of predictive biomarkers related to efficacy of immune checkpoint inhibitors, in combination with HPV status, might provide further clinical benefit to patients with HNSCC. 
Our study has several limitations. First, 33\% of patients diagnosed with OSCC were not included in the current analysis because of lack of tumor tissue, indicating a potential selection bias. Second, even though a relatively large cohort was analyzed, compared to previous studies, because of the favorable prognosis of OSCC only a few events occurred in the entire cohort. This might limit the power of the study to determine the prognostic role of PD-L1 expression. Consequently, it is not yet certain that PD-L1 expression truly has no prognostic role or it is just a reflection of lack of statistical power. Third, we did not explore PD-L1 expression in TILs. Finally, the inherent nature of a retrospective study makes it impossible to explore any dynamic changes in PD-L1 expression.

\section{Conclusion}

In conclusion, we showed that PD-L1 was highly expressed in OSCC, but there was no correlation between PD-L1 expression and HPV status. Further large prospective studies are required to determine the role of PD-L1 expression as a prognostic or predictive biomarker, and clinical studies of immune checkpoint inhibitors in OSCC are warranted regardless of HPV status.

\section{Conflicts of Interest}

Conflict of interest relevant to this article was not reported.

\section{References}

1. Gillison ML, Koch WM, Capone RB, Spafford M, Westra WH, $\mathrm{Wu} \mathrm{L}$, et al. Evidence for a causal association between human papillomavirus and a subset of head and neck cancers. J Natl Cancer Inst. 2000;92:709-20.

2. Marur S, D'Souza G, Westra WH, Forastiere AA. HPV-associated head and neck cancer: a virus-related cancer epidemic. Lancet Oncol. 2010;11:781-9.

3. Richards L. Human papillomavirus: a powerful predictor of survival in patients with oropharyngeal cancer. Nat Rev Clin Oncol. 2010;7:481.

4. Kreimer AR, Clifford GM, Boyle P, Franceschi S. Human papillomavirus types in head and neck squamous cell carcinomas worldwide: a systematic review. Cancer Epidemiol Biomarkers Prev. 2005;14:467-75.

5. Wiest T, Schwarz E, Enders C, Flechtenmacher C, Bosch FX. Involvement of intact HPV16 E6/E7 gene expression in head and neck cancers with unaltered $\mathrm{p} 53$ status and perturbed $\mathrm{pRb}$ cell cycle control. Oncogene. 2002;21:1510-7.

6. Bauman JE, Ferris RL. Integrating novel therapeutic monoclonal antibodies into the management of head and neck cancer. Cancer. 2014;120:624-32.

7. Flies DB, Sandler BJ, Sznol M, Chen L. Blockade of the B7H1/PD-1 pathway for cancer immunotherapy. Yale J Biol Med. 2011;84:409-21.

8. Robert C, Schachter J, Long GV, Arance A, Grob JJ, Mortier L, et al. Pembrolizumab versus ipilimumab in advanced melanoma. N Engl J Med. 2015;372:2521-32.

9. Garon EB, Rizvi NA, Hui R, Leighl N, Balmanoukian AS, Eder JP, et al. Pembrolizumab for the treatment of non-small-cell lung cancer. N Engl J Med. 2015;372:2018-28.

10. Seiwert TY, Burtness B, Weiss J, Gluck I, Eder JP, Pai SI, et al. A phase Ib study of MK-3475 in patients with human papillo- mavirus (HPV)-associated and non-HPV-associated head and neck (H/N) cancer. J Clin Oncol. 2014;32 Suppl:Abstr 6011.

11. Badoual C, Hans S, Merillon N, Van Ryswick C, Ravel P, Benhamouda $\mathrm{N}$, et al. PD-1-expressing tumor-infiltrating $\mathrm{T}$ cells are a favorable prognostic biomarker in HPV-associated head and neck cancer. Cancer Res. 2013;73:128-38.

12. Lyford-Pike S, Peng S, Young GD, Taube JM, Westra WH, Akpeng B, et al. Evidence for a role of the PD-1:PD-L1 pathway in immune resistance of HPV-associated head and neck squamous cell carcinoma. Cancer Res. 2013;73:1733-41.

13. Shi W, Kato H, Perez-Ordonez B, Pintilie M, Huang S, Hui A, et al. Comparative prognostic value of HPV16 E6 mRNA compared with in situ hybridization for human oropharyngeal squamous carcinoma. J Clin Oncol. 2009;27:6213-21.

14. Begum S, Gillison ML, Ansari-Lari MA, Shah K, Westra WH. Detection of human papillomavirus in cervical lymph nodes: a highly effective strategy for localizing site of tumor origin. Clin Cancer Res. 2003;9:6469-75.

15. Dong H, Zhu G, Tamada K, Flies DB, van Deursen JM, Chen L. B7-H1 determines accumulation and deletion of intrahepatic CD8(+) T lymphocytes. Immunity. 2004;20:327-36.

16. Gildener-Leapman N, Ferris RL, Bauman JE. Promising systemic immunotherapies in head and neck squamous cell carcinoma. Oral Oncol. 2013;49:1089-96.

17. Ukpo OC, Thorstad WL, Lewis JS Jr. B7-H1 expression model for immune evasion in human papillomavirus-related oropharyngeal squamous cell carcinoma. Head Neck Pathol. 2013;7: $113-21$.

18. Cho YA, Yoon HJ, Lee JI, Hong SP, Hong SD. Relationship between the expressions of PD-L1 and tumor-infiltrating lymphocytes in oral squamous cell carcinoma. Oral Oncol. 2011; 47:1148-53. 
19. Zhang F, Liu Z, Cui Y, Wang G, Cao P. The clinical significance of the expression of costimulatory molecule PD-L1 in nasopharyngeal carcinoma. Lin Chung Er Bi Yan Hou Tou Jing Wai Ke Za Zhi. 2008;22:408-10.

20. Hsu MC, Hsiao JR, Chang KC, Wu YH, Su IJ, Jin YT, et al. Increase of programmed death-1-expressing intratumoral CD8 $\mathrm{T}$ cells predicts a poor prognosis for nasopharyngeal carcinoma. Mod Pathol. 2010;23:1393-403.

21. Dong H, Strome SE, Salomao DR, Tamura H, Hirano F, Flies DB, et al. Tumor-associated B7-H1 promotes T-cell apoptosis: a potential mechanism of immune evasion. Nat Med. 2002; 8:793-800.

22. Kakavand H, Wilmott JS, Menzies AM, Vilain R, Haydu LE, Yearley JH, et al. PD-L1 expression and tumor-infiltrating lymphocytes define different subsets of MAPK inhibitor-treated melanoma patients. Clin Cancer Res. 2015;21:3140-8.

23. Bhandari M, Siva S. Re: Radical nephrectomy for pT1a renal masses may be associated with decreased overall survival compared with partial nephrectomy R. H. Thompson, S. A. Boorjian, C. M. Lohse, B. C. Leibovich, E. D. Kwon, J. C. Cheville and M. L. Blute J Urol 2008; 179: 468-473. J Urol. 2008; 180:1568-9.

24. Bellmunt J, Mullane SA, Werner L, Fay AP, Callea M, Leow JJ, et al. Association of PD-L1 expression on tumor-infiltrating mononuclear cells and overall survival in patients with urothelial carcinoma. Ann Oncol. 2015;26:812-7.

25. Westra WH. The changing face of head and neck cancer in the 21st century: the impact of HPV on the epidemiology and pathology of oral cancer. Head Neck Pathol. 2009;3:78-81. 